\title{
Successful Treatment of Recurrent Primitive Myxoid Mesenchymal Tumor of Infancy With BCOR Internal Tandem Duplication
}

\author{
Stuart L. Cramer, DO; ; Rong Li, MD, PhD ${ }^{\text {b; }}$ Siraj Ali, MD, PhDc; Julie A. Bradley, MD'; Hee K. Kim, MD;
} and Joseph G. Pressey, MD

\begin{abstract}
Primitive myxoid mesenchymal tumor of infancy (PMMTI) is a rare tumor with $<20$ cases reported to date. Recently PMMTI tumors have been found to harbor BCOR internal tandem duplication (ITD), the same genetic alteration detected in clear cell sarcoma of the kidney (CCSK). Complete surgical resection of PMMTI is often curative, but no standard of care has been established for unresectable tumors. We describe a female patient who presented at 13 months of age with a paraspinal mass and spinal cord compression. Histology was consistent with PMMTI, and the tumor was found to harbor BCOR ITD. The patient experienced disease recurrences after multiple surgical resections. After failing to respond to vincristine and actinomycin therapy, the patient demonstrated a nearly complete response to a doxorubicin-containing chemotherapy regimen. The patient's therapy was consolidated with proton beam radiotherapy, and she has remained in remission for $>12$ months after the conclusion of therapy. This case confirms BCOR ITD as a key finding in PMMTI. The therapeutic approach described here is similar to that used for CCSK and provides a model for the treatment of PMMTI not amenable to complete surgical resection.
\end{abstract}

Primitive myxoid mesenchymal tumor of infancy (PMMTI) is a rare fibroblastic-myofibroblastic malignancy that typically arises in the first year of life. Alaggio et $\mathrm{al}^{1}$ originally described PMMTI as being characterized by primitive spindle, polygonal, and round cells in a myxoid background with positive vimentin staining immunohistochemically, but negative for smooth muscle actin, muscle specific actin, desmin, myogenin, and S100 expression. Recently, internal tandem duplication (ITD) of BCOR has been detected in PMMTI, ${ }^{2}$ a molecular alteration identical to the hallmark feature found in most clear cell sarcoma of the kidney tumors (CCSK). ${ }^{3-6}$ The clinical course of PMMTI is often

From the aDepartment of Pediatrics, University of Alabama at Birmingham Birmingham, Alabama; 'Department of Pathology, Children's of Alabama, Birmingham, Alabama; 'Foundation Medicine, Cambridge, Massachusetts; ${ }^{d}$ Department of Radiation Oncology, University of Florida, Jacksonville, Florida; and 'Department of Radiology and ${ }^{f}$ Cancer and Blood Diseases Institute, Cincinnati Children's Hospital Medical Center, Cincinnati, Ohio. Submitted January 9, 2017; accepted for publication March 6, 2017. Dr. Ali has disclosed that he is an employee of and has equity in Foundation Medicine. The remaining authors have disclosed that they have no financial interests, arrangements, affiliations, or commercial indolent, with multiple recurrences reported in some patients. Treatment strategies have focused on surgical resection alone when feasible. No chemotherapy regimen has been established as a reliably effective standard of care for unresectable PMMTI tumors. Importantly, most patients with PMMTI with clinical follow-up reported to date $(n=19)$ were described as alive with disease $(n=10)$, dead of disease $(n=1)$, or dead with disease $(n=1)$ at most recent follow-up. Hence, the morbidity and mortality of this rare tumor type in young children is potentially immense. ${ }^{2,7}$ This report describes an infant with a recurrent PMMTI with confirmed BCOR

interests with the manufacturers of any products discussed in this article or their competitors.

Author contributions: Study concept and design: Cramer, Pressey. Pathologic evaluation of tumor: Li. Evaluation of radiologic findings: Kim. Data acquisition: Ali. Data analysis and interpretation: Ali. Manuscript preparation: Cramer, Li, Bradley, Kim, Pressey.

Correspondence: Joseph G. Pressey, MD, Cancer and Blood Diseases Institute, Cincinnati Children's Hospital Medical Center, 3333 Burnet Avenue, MLC 7013, Cincinnati, OH 45229.

E-mail: Joseph.Pressey@cchmc.org 
ITD that responded completely to multiagent chemotherapy, similar to regimens used successfully for CCSK.

\section{Methodology/Results}

A 13-month-old girl presented with progressive lower extremity weakness and bladder dysfunction. MRI revealed an epidural lesion extending from L3 to S2 level. The lesion showed low T1 and high T2 signal without contrast enhancement, suggesting a cystic lesion with neural foraminal extension. Initial radiologic diagnosis was an epidural arachnoid cyst (Figures 1 and 2). The patient underwent an L4 to L5 laminectomy with attempted total resection. Microscopically, the mass demonstrated a cellular neoplasm composed of undifferentiated-appearing stellate to epithelioid cells in an abundant myxoid matrix that stained diffusely positive for CD10 and vimentin, and weakly positive for bcl-2. These histologic findings were consistent with the diagnosis of PMMTI (Figure 3). Positive surgical margins were noted. Next-generation sequencing of the tumor revealed BCOR ITD as evidenced by a 30 amino acid in-frame duplicated sequence (S1705_S1706). ${ }^{8}$ Also identified was a mutation (Y325*) of CD36, which encodes a thrombospondin receptor and other genetic variations of unknown significance.
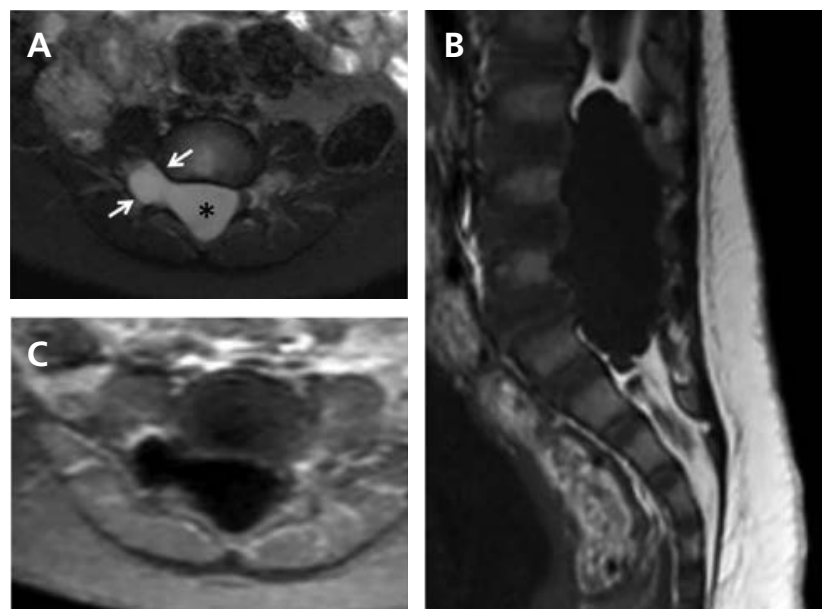

Figure 1. (A) Axial T2-weighted MRI with fat suppression at the time of diagnosis demonstrates an epidural cystic lesion (*) with neural foraminal extension (arrows). (B) Sagittal T1-weighted image shows a lobulated mass in the spinal canal extending from L3 to S2 level. (C) Postcontrast axial T1-weighted image with fat suppression shows thin peripheral enhancement without evidence of solid component. Initial diagnosis was arachnoid cyst.
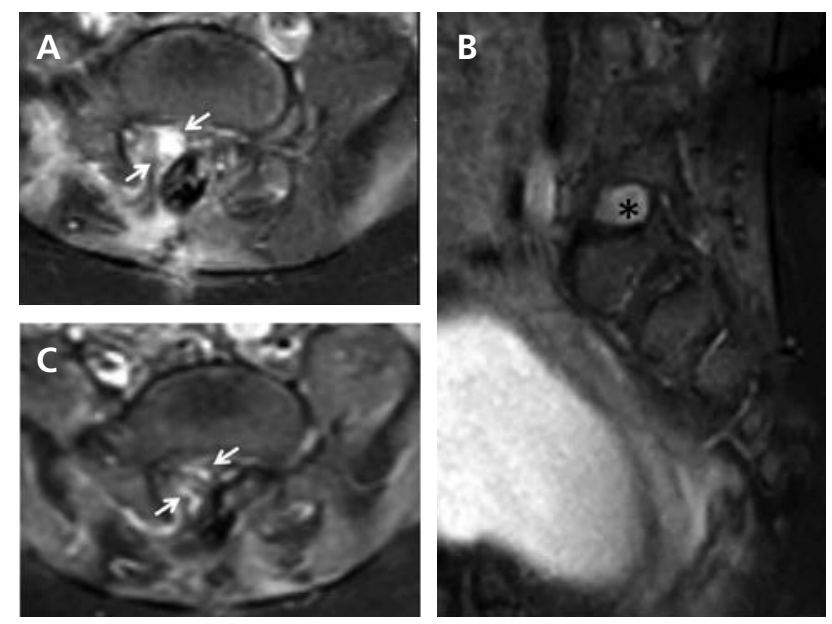

Figure 2. (A) Recurrent tumor demonstrated by postcontrast axial T1-weighted MRI with fat suppression showing postsurgical changes related to laminectomy and mass resection. There is a residual mass in the right epidural space at the level of L5-S1 spine. The lesion demonstrates diffuse enhancement (arrows). (B) Postcontrast sagittal T1-weighted image with fat suppression shows right L5-S1 neural foraminal extension $(*)$. (C) Postcontrast axial T1-weighted image with fat suppression shows near resolution of the right epidural mass with right neural foraminal extension following vincristine, doxorubicin, cyclophosphamide, ifosfamide, and etoposide therapy.

Two months after the initial diagnosis, a routine MRI revealed recurrence of the lumbar epidural mass. Over a 6-month period, the patient underwent 2 additional surgeries with positive microscopic margins; however, the tumor recurred within 8 weeks after each surgery. Due to the refractory nature of this tumor, the patient received 2 cycles of a chemotherapeutic regimen consisting of vincristine $(0.05$ $\mathrm{mg} / \mathrm{kg} /$ dose $)$ and actinomycin ( $0.045 \mathrm{mg} / \mathrm{kg} /$ dose $)$; however, MRI revealed continued tumor progression increasing from $0.8 \times 0.4 \mathrm{~cm}$ to $1.4 \times 1.0 \mathrm{~cm}$. At the age of 23 months, the patient then began vincristine $\left(1.5 \mathrm{mg} / \mathrm{m}^{2}\right)$, doxorubicin $\left(37.5 \mathrm{mg} / \mathrm{m}^{2} /\right.$ dose $\mathrm{x} 2$ days), and cyclophosphamide $\left(1,200 \mathrm{mg} / \mathrm{m}^{2}\right.$; VDC) therapy alternating with ifosfamide $\left(1,800 \mathrm{mg} / \mathrm{m}^{2} /\right.$ dose $\mathrm{x} 5$ days $)$ and etoposide $\left(100 \mathrm{mg} / \mathrm{m}^{2} /\right.$ dose $\mathrm{x} 5$ days; IE) on a 3-week interval. Imaging was performed after 2 courses of therapy (VDC and IE each given twice), with no discrete mass visible. The patient ultimately received a total of 3 courses each of VDC and IE. Therapy was consolidated with proton beam radiotherapy (RT) delivered in 2 phases, with the standard risk volume receiving 45 cobalt gray equivalent (CGE) and the volume deemed to be at highest risk receiving a total of 64.8 CGE using a 
3-field, 3-dimensional conformal proton plan. Special accommodations were used to ensure that the adjacent vertebral bodies received a uniform dose of 23.4 CGE to prevent growth asymmetry. The patient was documented to remain free of disease 12 months after completing therapy.

\section{Discussion}

The clinical features of the PMMTI described herein generally resemble those of previous reports, although the present patient was older than most at diagnosis. A subset of PMMTI cases have developed in the trunk, including 5 cases reported within the paraspinal region., ${ }^{2,7}$ Spinal cord compression at presentation has also been previously reported. ${ }^{1}$ The present case independently confirms the finding of BCOR ITD in PMMTI, providing key biological insight into the pathogenesis of this rare tumor type. The function of BCOR (BCL-6 corepressor) is to inhibit gene transcription by interacting with BCL-6, histone deacetylase (HDAC), demethylase, and H2A ubiquitin ligase. In addition, BCOR forms a complex with the polycomb group proteins and is a part of the noncanonical PRC1 complex. Based on this interaction, it is assumed that the overexpression and partial duplication of BCOR allows for tumorigenesis via an epigenetic pathway. ${ }^{5}$ Other BCOR alterations have been documented in a variety of sarcomas, including mutations found in rhabdomyosarcoma ${ }^{9}$ and chromosomal translocations in Ewing-like sarcoma (BCORCCND3), ${ }^{10}$ ossifying fibromyxoid tumor, and endometrial stromal sarcoma (ZC3H7B-BCOR). ${ }^{11}$

When feasible, complete resection of PMMTI is the preferred treatment; there are no reports of completely resected PMMTI tumors recurring. As demonstrated in the present case, local recurrences are common after incomplete resections. ${ }^{7}$ Most prior patients with incompletely resected tumors were alive with disease (5 of 7), one patient was without evident disease, and one patient was dead of disease at last follow-up.

Few data exist to guide the therapeutic approach for patients with unresectable PMMTI. Given the efficacy and tolerability of vincristine and actinomycin with or without cyclophosphamide (VAC) in infantile fibrosarcoma and embryonal rhabdomyosarcoma that are often encountered in infancy, such therapy would also seem appeal-

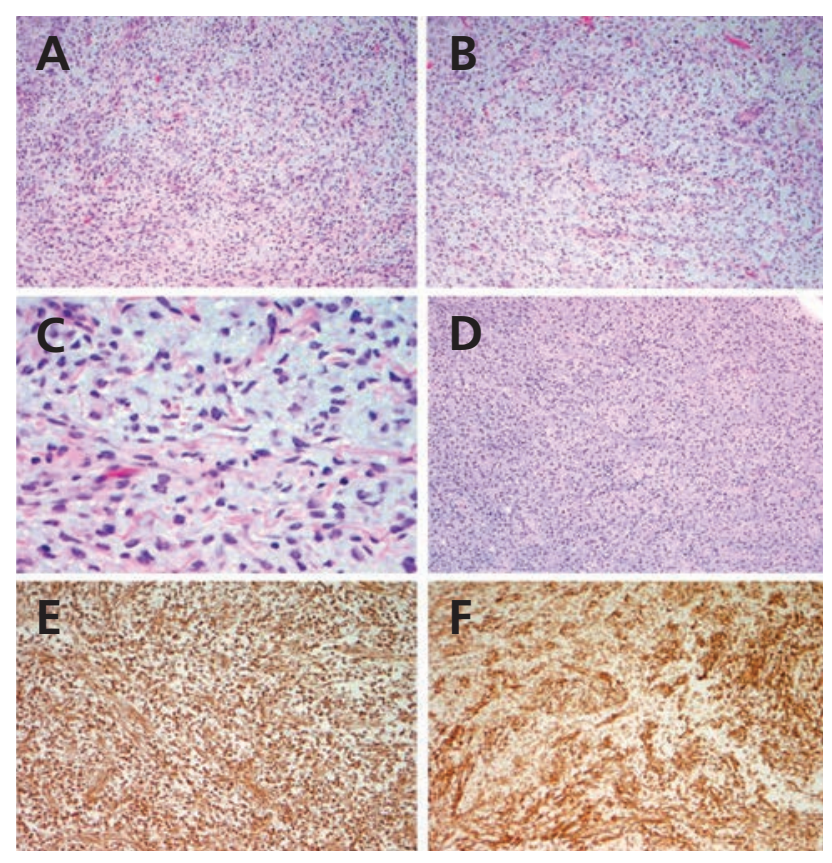

Figure 3. $(A, B)$ Microscopic examination of the tumor demonstrated a diffuse growth of primitive stellate to epithelioid cells in a myxoid background. There is no necrosis and mitotic figures are extremely rare. The tumor showed a well-developed, rich, vascular network with a lacy appearance seen in many areas (hematoxylin-eosin, original magnification $\times 100$ for both). (C) The tumor cells had bland, relatively uniform, nuclei with inconspicuous nucleoli, and variable amounts of pale eosinophilic to clear vacuolated cytoplasm (hematoxylin-eosin, original magnification $\times 400$ ). (D) The recurrent tumor displayed similar morphology with mildly increased cellularity (hematoxylin-eosin, original magnification $\times 100$ ). The tumor showed diffuse immunoreactivity for (E) vimentin and (F) CD10 (original magnification $\times 100$, for both).

ing for PMMTI. However, in addition to the present case treated with VA, 3 prior patients treated with VAC were reported to not respond, hence neither VAC nor VA can be considered effective for PMMTI based on currently available information. ${ }^{12-14}$ The use of ifosfamide and doxorubicin has been reported in 2 previous cases, with 1 partial response and 1 disease progression. ${ }^{12,13}$ Given the biological overlap between PMMTI and CCSK, treatment regimens used for CCSK may provide a template for PMMTI therapy. The role of doxorubicin-containing chemotherapy in CCSK is well established, ${ }^{15}$ and the Children's Oncology Group recently completed a trial using vincristine, doxorubicin, cyclophosphamide, and etoposide with RT for CCSK (ClinicalTrials.gov identifier: NCT00002611). Hence, the use of VDC/IE as was used successfully in the present case or a similar regimen is worthy of further investigation in PMMTI. As the role of $B C O R$ in malignant transformation 
is further elucidated, we anticipate that neoplasms driven by BCOR alterations will be found to be amenable to rationally designed targeted therapies. ${ }^{16}$

Yet, although the patient responded well to this regimen and only experienced commonly expected toxicities, the potential for cardiomyopathy, secondary cancers, and infertility is significant and must be taken into consideration. Likewise, RT carries potentially dramatic morbidity in infants, but may be necessary in cases where local control cannot be effectively achieved with surgery alone. In the present case, the tumor recurred after multiple attempted complete surgical resections. Despite the subsequent response to chemotherapy, definitive local control was still deemed necessary. Thus, the patient's therapy was consolidated with proton beam RT, an attractive option for infantile sarcomas adjacent to radiosensitive healthy tissues. ${ }^{17}$

\section{References}

1. Alaggio R, Ninfo V, Rosolen A, et al. Primitive myxoid mesenchymal tumor of infancy: a clinicopathologic report of 6 cases. Am J Surg Pathol 2006;30:388-394

2. Kao YC, Sung YS, Zhang L, et al. Recurrent BCOR internal tandem duplication and YWHAE-NUTM2B fusions in soft tissue undifferentiated round cell sarcoma of infancy: overlapping genetic features with clear cell sarcoma of kidney. Am J Surg Pathol 2016;40:1009-1020.

3. Karlsson J, Valind A, Gisselsson D. BCOR internal tandem duplication and YWHAE-NUTM2B/E fusion are mutually exclusive events in clear cell sarcoma of the kidney. Genes Chromosomes Cancer 2016;55:120-123.

4. Kenny C, Bausenwein S, Lazaro A, et al. Mutually exclusive BCOR internal tandem duplications and YWHAE-NUTM2 fusions in clear cell sarcoma of kidney: not the full story. J Pathol 2016;238:617-620.

5. Ueno-Yokohata H, Okita $\mathrm{H}$, Nakasato $\mathrm{K}$, et al. Consistent in-frame internal tandem duplications of BCOR characterize clear cell sarcoma of the kidney. Nat Genet 2015;47:861-863.

6. Roy A, Kumar V, Zorman B, et al. Recurrent internal tandem duplications of BCOR in clear cell sarcoma of the kidney. Nat Commun 2015;6:8891.

7. Foster JH, Vasudevan SA, John Hicks M, et al. Primitive myxoid mesenchymal tumor of infancy involving chest wall in an infant: a case report and clinicopathologic correlation. Pediatr Dev Pathol 2016;19:244248.

8. Frampton GM, Fichtenholtz A, Otto GA, et al. Development and validation of a clinical cancer genomic profiling test based on massively parallel DNA sequencing. Nat Biotechnol 2013;31:1023-1031.

9. Shern JF, Chen L, Chmielecki J, et al. Comprehensive genomic analysis of rhabdomyosarcoma reveals a landscape of alterations affecting a common genetic axis in fusion-positive and fusion-negative tumors. Cancer Discov 2014;4:216-231.

10. Pierron G, Tirode F, Lucchesi C, et al. A new subtype of bone sarcoma defined by BCOR-CCNB3 gene fusion. Nat Genet 2012;44:461-466.

11. Antonescu CR, Sung YS, Chen CL, et al. Novel ZC3H7B-BCOR, MEAF6-PHF1, and EPC1-PHF1 fusions in ossifying fibromyxoid tumorsmolecular characterization shows genetic overlap with endometrial stromal sarcoma. Genes Chromosomes Cancer 2014;53:183-193.

12. Cuthbertson DW, Caceres K, Hicks J, et al. A cooperative approach to diagnosis of rare diseases: primitive myxoid mesenchymal tumor of infancy. Ann Clin Lab Sci 2014;44:310-316.

13. Guilbert MC, Rougemont AL, Samson Y, et al. Transformation of a primitive myxoid mesenchymal tumor of infancy to an undifferentiated sarcoma: a first reported case. J Pediatr Hematol Oncol 2015;37:e118-120.

14. Mulligan L, O'Meara A, Orr D, et al. Primitive myxoid mesenchymal tumor of infancy: a report of a further case with locally aggressive behavior. Pediatr Dev Pathol 2011;14:75-79.

15. Green DM, Breslow NE, Beckwith JB, et al. Treatment of children with clear-cell sarcoma of the kidney: a report from the National Wilms' Tumor Study Group. J Clin Oncol 1994;12:2132-2137.

16. Beguelin W, Teater M, Gearhart MD, et al. EZH2 and BCL6 cooperate to assemble CBX8-BCOR complex to repress bivalent promoters, mediate germinal center formation and lymphomagenesis. Cancer Cell 2016;30:197-213.

17. Ladra MM, Szymonifka JD, Mahajan A, et al. Preliminary results of a phase II trial of proton radiotherapy for pediatric rhabdomyosarcoma. J Clin Oncol 2014;32:3762-3770.

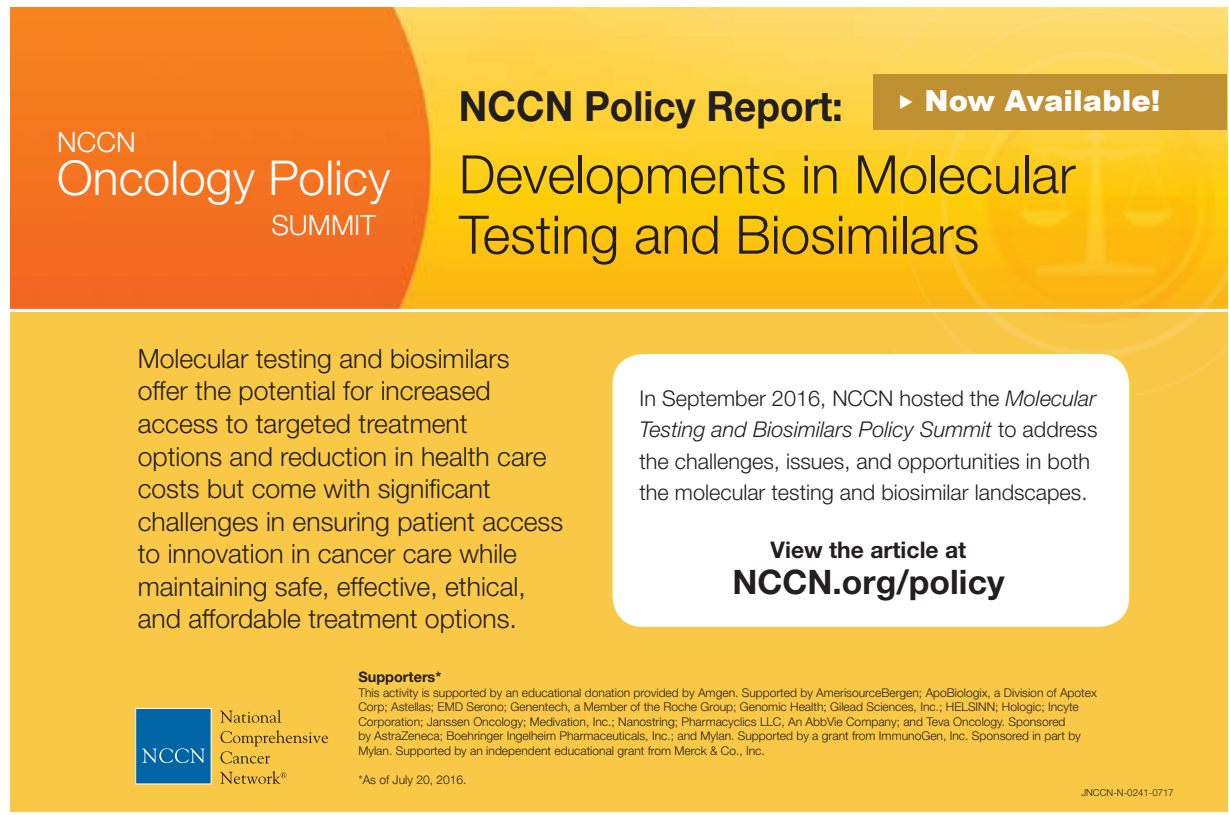

